\title{
Conventions and abbreviations
}

Example sentences consist of four lines. The first line uses a practical orthography. The second line shows the underlying morphemes. The third line is a gloss, and the fourth line provides a free translation in English. In glosses, proper names are represented by initials. Constituents are in square brackets. Examples are numbered separately for each chapter. If elements are marked in other ways (e.g. with boldface), this is indicated where relevant. A source reference is given for each example; the reference code consists of the initials of the speaker (or 'Game' for the Man and Tree games), the date the recording was made, and the number of the utterance. An overview of recordings plus reference codes can be found in Appendix I.

The following abbreviations and symbols are used in glosses:

\begin{tabular}{ll}
1 & first person \\
2 & second person \\
3 & third person \\
ADJ & adjectiviser \\
ANIM & animate \\
APPL & applicative \\
CAUS & causative \\
CLF & classifier \\
CNTF & counterfactual \\
COMP & complementiser \\
COND & conditional \\
CONT & continuative \\
DEF & definiteness marker \\
DEM & demonstrative \\
DIM & diminutive \\
DIST & distal \\
du & dual \\
EMP & emphatic marker \\
EXCL & exclusive \\
FREE & free pronoun \\
FOC & focus marker \\
HAB & habitual \\
IMP & imperative \\
INANIM & inanimate \\
INCL & inclusive \\
INT & intermediate \\
INTJ & interjection \\
INTF & intensifier \\
IPFV & imperfective \\
IRR & irrealis \\
MOD & modal operator \\
NEG & negation marker \\
NOM & nominaliser \\
NS & non-singular \\
PART & partitive \\
& \\
\hline
\end{tabular}




$\begin{array}{ll}\text { PC } & \text { paucal } \\ \text { PERT } & \text { pertensive } \\ \text { PFV } & \text { perfective } \\ \text { pl } & \text { plural } \\ \text { POSS } & \text { possessive } \\ \text { PRF } & \text { perfect } \\ \text { PROG } & \text { progressive } \\ \text { PROX } & \text { proximate } \\ \text { RECIP } & \text { reciprocal } \\ \text { REDUP } & \text { reduplication } \\ \text { REL } & \text { relative clause marker } \\ \text { Sg } & \text { singular } \\ \text { SPEC.COLL } & \text { specific collective } \\ \text { SUB } & \text { subordinate clause marker } \\ \text { TAG } & \text { tag question marker } \\ \text { ZERO } & \text { person/number reference with no overt realisation } \\ - & \text { affix boundary } \\ = & \text { clitic boundary } \\ \text {. } & \text { morpheme boundary }\end{array}$

The following abbreviations are used as subscripts to constituents:

$\begin{array}{ll}\text { A } & \text { transitive subject } \\ \text { AdvCl } & \text { adverbial subordinate clause } \\ \text { AG } & \begin{array}{l}\text { animate goal } \\ \text { AM }\end{array} \\ \text { associated motion } \\ \text { Apo } & \text { apodosis } \\ \text { BEN } & \text { benefactive } \\ \text { CC } & \text { copula complement } \\ \text { Compl } & \text { complement clause } \\ \text { E } & \text { extended argument } \\ \text { LG } & \text { locative goal } \\ \text { LOC } & \text { locative } \\ \text { MAL } & \text { malefactive } \\ \text { MC } & \text { main clause } \\ \text { NVPRE } & \text { non-verbal predicate } \\ \text { O } & \text { transitive object } \\ \text { Obl } & \text { oblique argument } \\ \text { Pot } & \text { potential } \\ \text { Pro } & \text { protasis } \\ \text { Quot } & \text { quotative } \\ \text { RC } & \text { relative clause } \\ \text { S } & \text { intransitive subject } \\ \text { SVC } & \text { serial verb construction } \\ \text { Top } & \text { topicalised element }\end{array}$


The following abbreviations are used in running text and in tables:

$\begin{array}{ll}\text { A } & \text { adjective } \\ \text { Adv } & \text { adverb } \\ \text { CA } & \text { common argument } \\ \text { CoreAsp } & \text { core aspect } \\ \text { DC } & \text { deictic centre } \\ \text { DIR } & \text { directional } \\ \text { FC } & \text { focal clause } \\ \text { FoR } & \text { frame of reference } \\ \text { intr } & \text { intransitive } \\ \text { LG } & \text { locative goal } \\ \text { Manip } & \text { implement argument of ditransitive AFFECT verb } \\ \text { Mod } & \text { modifier } \\ \text { N, NP } & \text { noun, noun phrase } \\ \text { n/a } & \text { not applicable } \\ \text { POc } & \text { Proto-Oceanic } \\ \text { ProObj } & \text { object bound pronoun } \\ \text { ProSubj } & \text { subject bound pronoun } \\ \text { PV } & \text { postverbal element } \\ \text { S.b. } & \text { somebody } \\ \text { SC } & \text { supporting clause } \\ \text { SecAsp } & \text { secondary aspect } \\ \text { S.t. } & \text { something } \\ \text { Stat } & \text { stative } \\ \text { SVC } & \text { serial verb construction } \\ \text { TAM } & \text { Tense-Aspect-Modality } \\ \text { tr } & \text { transitive } \\ \text { V } & \text { verb } \\ \text { VC } & \text { verb complex } \\ & \end{array}$


